\title{
Depresión y cólera-hostilidad en alumnos víctimas de violencia intrafamiliar, de cuarto grado de secundaria de instituciones educativas estatales de zonas urbano marginales de la ciudad de Lima
}

Depression and anger-hostility in fourth -high school- grade students victims of domestic violence in high public educational institutions from urban marginal zones in the city of Lima

Rosa Huerta R. ${ }^{1}$, Jaime Aliaga., Emma Campos, Natalia Ramirez, Renato Santiváñez, Jessica Ramos, Lourdes Murillo, César Falcón, Nohely Treneman, María Tejada, Miriam Castro, Pedro Pérez

Universidad Nacional Mayor de San Marcos, Lima, Perú

(RECIBIDo 09/06/2015, AcEPTAdo 15/11/2015)

\section{RESUMEN}

La depresión es un trastorno del estado de ánimo prevalente en nuestro país que al presentarse en la niñez o en la adolescencia aumenta la vulnerabilidad a sufrir desórdenes afectivos en la etapa adulta, con claras repercusiones para el funcionamiento psicosocial de la persona. Asimismo la cólera-hostilidad trae consigo implicancias negativas para la salud, y en muchos casos, predice situaciones de violencia, pues es visto como una consecuencia de un mal manejo de las emociones.

En el estudio se sometió al análisis las variables depresión y cólera-hostilidad en función a la presencia o no de violencia intrafamiliar en una muestra de 472 alumnos de cuarto grado de secundaria víctimas y no víctimas de violencia intrafamiliar, a quienes se les administró una ficha de datos sociodemográficos, el Cuestionario de Depresión infantil y el Inventario Multicultural de Cólera-Hostilidad, integrada por los componentes Cólera Estado (CE), Cólera Rasgo (CR) y Expresión de Cólera. Se encontró que existe una correlación muy significativa entre la depresión con cada uno de los componentes de Cólera-Hostilidad, es decir con CE, CR y con Expresión de Cólera en los adolescentes víctimas de violencia intrafamiliar (sig. =0.00). Además, que las mujeres presentan mayor depresión en la muestra general, cuando hay violencia intrafamiliar son las mujeres las que presentan mayor depresión. Asimismo se halló una correlación significativa entre depresión con CE y CR con respecto a toda la muestra. Es la presencia de violencia intrafamiliar la que determina y produce trastornos en la salud mental de los adolescentes.

Palabras clave: Depresión, cólera, hostilidad, adolescentes, violencia intrafamiliar.

1 Docente Asociada de la Facultad de Psicología de la UNMSM. E-mail: huertarosel@hotmail.com 
DEPRESIÓN Y CÓLERA-HOSTILIDAD EN ALUMNOS VICTIMAS DE VIOLENCIA INTRAFAMILIAR...

\begin{abstract}
Depression is a mood disorder prevalent in our country that increases vulnerability to suffer affective disorders in adulthood when it appears in childhood or adolescence, with clear implications for the psychosocial functioning. Furthermore anger-hostility brings negative health implications, and in many cases, predicts situations of violence, for it is seen as a result of poor emotional control.

The study was subjected to the analysis of depression and anger-hostility variables according to the presence or not of domestic violence in a sample of 472 fourth high school gradestudents, victims and non-victims of domestic violence, who were given a sociodemographic data survey, the Children's Depression Inventory and the Multicultural State-Trait Anger Expression Inventory, consisting of Wrath State (CE), Trait Anger (CR) and Expression of Anger components. It was found that there is a significant correlation between depression with each of the components of Anger-Hostility, specifically with CE, CR and Expression of anger in adolescents victims of domestic violence $($ sig. $=0.00)$. In addition, women present more depressive episodes in general, when there is domestic violence, women use to present higher depressive levels. A significant correlation between depression with CE and $\mathrm{CR}$ with respect to the entire sample was also found. It is the presence of domestic violence that determines and produces mental disorders in adolescent health.
\end{abstract}

Keywords: Depression, anger, hostility, adolescents, domestic violence.

\title{
INTRODUCCIÓN
}

Los estudios sobre depresión y su relación con la cólera-hostilidad en la adolescencia, ponen de manifiesto las consecuencias de la violencia intrafamiliar en la salud mental de esta etapa de vida, con una alta prevalencia para la depresión, 8.6\% en adolescentes en Lima Metropolitana y Callao (ISM HD-IN, 2007), el cual asociado a la emoción de cólera-hostilidad aumenta la probabilidad de conducta violenta. Por ello Spielberger y Reheiser (2009), consideran que las manifestaciones de ansiedad, depresión y cólera son signos psicológicos vitales que están fuertemente relacionados de forma negativa con el bienestar de los individuos. Por ello es importante tomar en cuenta las variaciones, duración e intensidad de estas emociones en la vida diaria de los adolescentes con el fin de obtener información sobre su estado de salud mental.

La depresión, de acuerdo a diversos estudios, indica la importancia que tiene el ambiente familiar para el desarrollo de este trastorno. Entre los principales aspectos que han sido identificados como factores de riesgo, destacan la presencia de problemas de comunicación entre padres e hijos (Estévez, Musitu y Herrero, 2005; Jiménez et al., 1999) y la percepción de los adolescentes acerca de que en su familia existe un alto nivel de conflictividad, hostilidad y rechazo, (Villatoro, Andrade, Fleiz, Medina-Mora, Reyes y Rivera, 1997). Datos que el Ministerio de Salud (2009), en el estudio denominado Análisis de Situación de Salud de las y los Adolescentes, detectó entre los problemas psicosociales, que los principales factores por los cuales las y los adolescentes intentan suicidarse, son los mismos tanto para la población de la costa, sierra y selva, siendo el principal motivo los 
Rosa Huerta, Jaime Aliaga, Emma Campos, Natalia Ramírez, Renato Santiváñez, Jessica Ramos, Lourdes Murillo,

César Falcón, Nohely Treneman, María Tejada, Miriam Castro, Pedro Pérez

problemas con los padres. Es así que la familia es el factor protector y puede convertirse en factor de riesgo cuando surgen problemas dentro de ella.

Frente a esta problemática en el presente estudio se exploran las variables depresión y cólera-hostilidad en una muestra de 472 adolescentes de cuarto grado de secundaria de Instituciones Educativas Estatales, víctimas y no víctimas de violencia intrafamiliar a quienes además se les administra una ficha de datos sociodemográficos, con el objetivo de estudiar la relación entre la presencia de depresión con la cólera-hostilidad, en ambos grupos en función al sexo y comprobar si la presencia de ambas variables, están vinculadas con la situación de violencia familiar.

La adolescencia como etapa de vida es la población comprendida desde los 12 años de edad hasta los 17 años, 11 meses y 29 días, (bajo Resolución Ministerial N. ${ }^{\circ}$ 538-2009/MINSA, 14 de agosto del 2009). En el Perú la población adolescente de 12 a 18 años, representa el $13.11 \%$ de la población total (Sistema de focalización de hogares, INEI. 2012-2013) porcentaje significativo que amerita poner atención a este grupo de edad. Ahora bien, desde la perspectiva del adolescente, esta etapa de vida presenta cambios difíciles que les producen ansiedad, confusión, desorientación y hasta ideas de suicidio, sumado a ello la ausencia de calidez familiar, falta de comunicación con los padres y discordia familiar, hacen que sea disfuncional y existan oportunidades limitadas para el aprendizaje de resolución de problemas y pueden crear un ambiente donde al adolescente le falta el soporte necesario para contrarrestar los efectos de estos eventos vitales.

En el estudio realizado por Vargas, Tovar y Velarde (2010) sobre Prevalencia y factores asociados con el episodio depresivo en adolescentes de Lima Metropolitana y Callao encontraron una prevalencia actual del episodio depresivo en adolescentes de $8.6 \%$. Alcanzando una mayor prevalencia de episodio depresivo en el grupo etario de 15 a 17 años (12.1\%), en comparación con el grupo etario de 12 a 14 años $(5.4 \%)$. De la misma forma, la prevalencia fue mayor en el sexo femenino (13.4\%), que en el masculino (3.9\%); asimismo, en cuanto a la prevalencia de episodio depresivo en adolescentes según sensación de soporte familiar y social, se encontró que, si esta disminuía la prevalencia de episodio depresivo aumentaba (de $6.2 \%$ a $14 \%$ ). En cuanto a relaciones intrafamiliares, algunos estudios han encontrado que la depresión en adolescentes emerge y puede ser reforzada dentro del contexto de patrones de relación familiar disruptivos o perturbados, incluyendo pobre comunicación, rechazo, desaprobación parental y baja cohesión familiar (INSMHD-HN, 2002).

Por otro lado, la prevalencia de vida de conductas agresivas en los adolescentes, como peleas con algún tipo de armas, abuso físico a menores, se halló un 23,5\% (INSM HD-HN, 2002). Ello nos permite observar la importancia de estudiar la presentación de cólera-hostilidad, al respecto Spielberger (1993) establece el síndrome AHA (Anger-Hostility-Agression), en donde la ira es un estado emocional que puede llegar a generar hostilidad. Cuando esta hostilidad se vuelve visible, se presenta como comportamiento agresivo, que tiene la intención de destruir o atacar aquello que lo generó, es pues un estado emocional que afecta el desarrollo del 
adolescente especialmente de aquellos inmersos dentro de familias donde existe violencia. Además de las consecuencias directas, como varios estudios encuentran, los hijos tienden a repetir los patrones de violencia vividos en sus hogares. Los padres actúan como modelos de comportamientos violentos que los hijos observan y luego imitan, confirmándose el hecho de que existe la transmisión intergeneracional de la violencia vivida en los hogares; también se ha encontrado que los jóvenes con una historia de victimización son más propensos a comprometerse en una variedad de comportamientos riesgosos. Así estudios en Lima como el de Chapi (2012) encuentra una relación inversa entre la satisfacción familiar y la cólera estado y cólera rasgo observándose que la percepción de satisfacción o insatisfacción familiar inciden en la expresión de cólera, es decir ante el incremento de satisfacción familiar en los adolescentes varones y mujeres, disminuirá la cólera que forma parte del rasgo de personalidad y viceversa.

\section{Violencia intrafamiliar y adolescencia}

El maltrato hacia niñas/os y adolescentes, se refiere a las distintas formas de relación abusiva que caracteriza de modo permanente o cíclico el vínculo intrafamiliar. El término violencia familiar, alude a todas las formas de violencia que tienen lugar en las relaciones entre los miembros de una familia. Se denomina relación de abuso a aquella forma de interacción que, enmarcada en un contexto de desequilibrio de poder, incluye conductas de una de las partes que, por acción u omisión, ocasionan daño físico y/o psicológico a otro miembro de la relación (Corsi, 2000).

Precisamente la familia, contrariamente a lo que siempre se nos ha enseñado que es una fuente de calor, afecto y seguridad, pasa a ser el lugar más que propicio para el surgimiento de situaciones de violencia en su interior, Huerta (2014) encontró que la violencia entre los progenitores que tienen como testigos permanentes a los niños y adolescentes, es más un problema relacional que un problema estructural. Es necesario subrayar que para definir una situación familiar como un caso de violencia intrafamiliar, la relación de abuso debe ser crónica, permanente o periódica

Diariamente observamos que numerosos adolescentes, sufren las secuelas de la violencia intrafamiliar, y con demasiada frecuencia se encuentran acompañadas por el silencio de su entorno social. Son miles de menores, adolescentes, las víctimas cotidianas de la violencia familiar, en todas sus modalidades: psicológica, física y sexual; víctimas que en su gran mayoría sufren silenciosamente la crueldad de sus padres y familiares, y que probablemente ya han aprendido y naturalizado tanto la violencia que se les hace imposible visualizar la violencia vivida y la experimentan como algo natural.

Cuando la violencia ha sido lo suficientemente crónica y extrema, las repercusiones se empiezan a manifestar en las aulas escolares ya sea con conductas de aislamiento, agresión hacia sus compañeros (bullying), maestros o a veces se detecta como bajo rendimiento escolar o fracaso escolar (Muro, 2009). 
Rosa Huerta, Jaime Aliaga, Emma Campos, Natalia Ramírez, Renato Santiváñez, Jessica Ramos, Lourdes Murillo,

César Falcón, Nohely Treneman, María Tejada, Miriam Castro, Pedro Pérez

Desde la perspectiva de un niño o adolescente, el maltrato recibido de sus padres o cuidadores constituye un tipo de violencia directa, mientras que ser testigo de la violencia entre sus padres conforma un tipo de violencia indirecta y que tiene secuelas importantes, entre ellas apartarse emocionalmente de su familia o sus amigos, volverse agresivos o enojados, sentirse deprimidos o deprimidas, involucrarse en conductas de riesgo.

\section{Depresión}

La depresión en adolescentes es una condición común, crónica, recurrente y de tendencia familiar que usualmente persiste en la adultez. Se considera la posibilidad de que aquella que se inicia en la infancia o la adolescencia sea una forma más severa del trastorno (Vargas, H. et al; 2010).

Actualmente, la depresión en adolescentes se puede diagnosticar siguiendo los mismos criterios usados en los adultos. Para hacer el diagnóstico se pueden utilizar los sistemas de clasificación más empleados, como el DSM IV-TR y la Clasificación Internacional de Enfermedades (CIE-10). En términos generales, según el DSM IV-TR, la depresión puede dividirse en depresión mayor, distimia y trastorno depresivo recurrente. En la CIE-10 no se emplea el término "depresión mayor" y se describe un cuadro equivalente llamado "episodio depresivo" (cuando hay un solo episodio) o trastorno depresivo recurrente (cuando hay más de un episodio).

Diferentes estudios a lo largo de los años, han estimado una prevalencia de depresión mayor (equivalente al episodio depresivo) en adolescentes que fluctúa entre $0.4 \%$ a $8.3 \%$ y una prevalencia de vida entre $15 \%$ y $20 \%$ (INSM HD-HN, 2002). Estudios clínicos y epidemiológicos reportan una mayor prevalencia en adolescentes mujeres que en varones. Por otro lado, la prevalencia de episodio depresivo en adolescentes víctimas de violencia intrafamiliar, mostró resultados diversos. Los adolescentes que fueron víctimas de algún tipo de abuso (físico, psicológico, sexual o negligencia) presentaban una mayor prevalencia de episodio depresivo (13.1\%), en comparación con los que no tenían ningún tipo de abuso (3.9\%) (Vargas, H. et al; 2010).

La adolescencia como etapa de vida, es uno de los grupos etarios que presenta mayor probabilidad de sufrir depresión, ya que en esta etapa se llevan a cabo procesos de cambio físico, psicológico, sociocultural y cognitivo, que demandan de los jóvenes el desarrollo de estrategias de afrontamiento que les permitan establecer un sentido de identidad, autonomía y éxito personal y social (Blum, 2000). Además, la adolescencia es el periodo de desarrollo más importante para la adquisición y el mantenimiento de patrones de comportamiento saludable que disminuyan el riesgo del desarrollo de trastornos clínicos durante este periodo y la edad adulta. (Lewinsohn, Rohde, Seeley, Klein \& Gotlib, 2000).

Entre los factores de riesgo para presentar depresión se encuentran, haber sido víctima de abuso o maltrato infantil, tanto físico como sexual, enfermedad crónica, antecedentes familiares de depresión, deficientes habilidades sociales así como 
acontecimientos estresantes de la vida. En el caso de las mujeres, que presentaron mayores sucesos estresantes tienen 3.6 mayor puntaje de síntomas de depresión cuando fueron comparadas con los hombres.

La Guía técnica para el reconocimiento de la depresión en niños y adolescentes del Ministerio de Salud (2007) señala que deben presentarse cinco de los síntomas que se detallan en la Tabla 1, en tanto interfieran con su vida diaria por un periodo mínimo de dos semanas.

Tabla 1. Reconocimiento y diagnóstico de la depresión en niños/adolescentes

\begin{tabular}{l}
\hline COMO SE PUEDE OBSERVAR EN LOS JÓVENES \\
\hline Estado de ánimo irritable o malhumorado, preocupación con letras nihilistas de canciones. \\
Pérdida del interés en los deportes, video juegos, y en actividades con amigos. \\
Fracaso en el aumento de peso como normalmente se espera, anorexia o bulimia; quejas frecuentes \\
sobre enfermedades físicas, por ejemplo dolor de cabeza o de estómago. \\
Mirar la televisión hasta altas horas de la noche, negación a levantarse para asistir a la escuela \\
en la mañana. \\
Hablar acerca de huir de casa o realizar esfuerzos para huir. \\
Aburrimiento persistente. \\
Comportamiento opuesto o negativo. \\
Bajo rendimiento en la escuela, frecuentes faltas. \\
Ideas o comportamientos suicidas recurrentes.
\end{tabular}

\section{Cólera-Hostilidad}

Spielberger (1972, citado por Moscoso, 1998), definió la cólera como un estado emocional, el cual consiste en sentimientos que varían de intensidad, desde una ligera irritación o molestia hasta la experimentación de una furia intensa y episodios de rabia. Agrega además, que se suele citar los términos cólera, enojo e ira de forma intercambiables.

Dentro de las principales definiciones del concepto cólera, encontramos la de Izard (Izard, 1991), quien la describe como una respuesta emocional primaria, ya que surge de forma automática ante determinado evento como producto de una evaluación cognitiva de amenaza. De igual forma, Reeve (2001) la define como una reacción emocional primaria y negativa, caracterizada por la irritación, furia o gran enfado, causada por la indignación y el enojo de sentir vulnerados nuestros derechos. Asimismo, en situaciones muy extremas, puede generar conductas de violencia y reacciones de odio, lo cual puede ocasionar que sea muy destructiva en caso de que no se maneje de forma adecuada (Fernández Abascal, Martín y Domínguez, 2001).

Por otro lado, en cuanto al uso de los términos enojo o cólera y hostilidad se suele confundir. Es por ello importante diferenciarlos; la hostilidad se encuentra 
Rosa Huerta, Jaime Aliaga, Emma Campos, Natalia Ramírez, Renato Santiváñez, Jessica Ramos, Lourdes Murillo,

César Falcón, Nohely Treneman, María Tejada, Miriam Castro, Pedro Pérez

más relacionada a un conjunto de actitudes las cuales suelen motivar conductas agresivas que se encuentran dirigidas a causar daño a objetos o personas. Es decir, la cólera hace referencia a sentimientos, y la hostilidad hacia actitudes, relacionadas en ocasiones con conductas violentas hacia otros. Por su parte, el concepto de agresión por lo general suele implicar la conducta en sí, punitiva o destructiva hacia otras.

Habitualmente el adolescente agresivo se ve a sí mismo en un mundo amenazante. Sus experiencias dolorosas, muchas veces de violencia intrafamiliar, de abuso y abandono le han enseñado que el entorno es hostil. En él predominan la rigidez en el pensamiento, incapacidad para la abstracción y fantasías focalizadas sobre la violencia. Así los pensamientos y emociones relacionados con la cólera aparecen y se interrelacionan con la experiencia y los aprendizajes pasados del sujeto, y se agrava cuando vive dentro de un ambiente de violencia familiar. Como también lo encuentra Chapi (2012), en un estudio con adolescentes, que a mayor satisfacción familiar, menores indicadores de ansiedad y de cólera-hostilidad, a la vez mayor control de emociones coléricas. Ello nos lleva a manifestar que la familia en la cual están insertas las y los adolescentes es relevante en su desarrollo como etapa de vida y en su salud mental.

\section{HIPÓTESIS}

H1: Existe una relación positiva entre la depresión y la expresión de cólerahostilidad en alumnos de cuarto grado de secundaria con y sin violencia intrafamiliar de Instituciones Educativas estatales de zonas urbano marginales de la ciudad de Lima.

H2: Existen diferencias estadísticamente significativas en la presencia de depresión y cólera-hostilidad según el sexo en alumnos de cuarto grado de secundaria con y sin violencia intrafamiliar de Instituciones Educativas estatales de zonas urbano marginales de la ciudad de Lima.

H3: Existen diferencias estadísticamente significativas en los niveles de depresión entre los alumnos con y sin violencia intrafamiliar de Instituciones Educativas estatales de zonas urbano marginales de la ciudad de Lima.

H4: Existen diferencias estadísticamente significativas en los tipos de expresión de cólera entre los alumnos con y sin violencia intrafamiliar de Instituciones Educativas estatales de zonas urbano marginales de la ciudad de Lima.

\section{MÉTODO}

El presente estudio es de tipo sustantivo, que sistematiza procedimientos dentro del método correlacional y adicionalmente de la contrastación que nos permitió conocer tanto la intensidad y dirección de la relación entre la depresión y la cólera-hostilidad en adolescentes de cuarto grado de secundaria con y sin violencia intrafamiliar 
DEPRESIÓN Y CÓLERA-HOSTILIDAD EN ALUMNOS VICTIMAS DE VIOLENCIA INTRAFAMILIAR...

según el sexo. El diseño fue descriptivo correlacional, transversal, los datos fueron recogidos en un solo momento (Hernández, Fernandez y Baptista, 2010).

\section{Muestra}

La muestra quedó constituida por 472 alumnos hombres y mujeres de Instituciones Educativas estatales de zonas urbano marginales de la ciudad de Lima, que se fueron clasificando según edad, por la presencia o no de violencia, por el tipo de violencia. Aplicándose un muestreo no probabilístico, intencional; se seleccionaron las instituciones educativas más representativas para su evaluación. Aplicándose los instrumentos de manera grupal.

La edades que presentaron los y las adolescentes fueron entre 14 y 16 años predominando la edad de 15 a 16 años tanto en el grupo con violencia como el de sin violencia. En cuanto a la muestra por grupo víctima y no víctima de violencia intrafamiliar según sexo, se encontró mayor porcentaje, tanto en mujeres (37.29\%) como en hombres $(35.59 \%)$ de pertenencia al grupo víctima de violencia.

Tabla 2. Distribución muestral por grupo víctima y no víctima de violencia familiar y el sexo.

\begin{tabular}{cccc}
\hline Sexo & Víctima de violencia & No víctima de violencia & Total \\
\hline Mujer & 176 & 60 & 236 \\
& $37.29 \%$ & $12.71 \%$ & $50.00 \%$ \\
Hombre & 168 & 68 & 236 \\
& $35.59 \%$ & $14.41 \%$ & $50.00 \%$ \\
\hline Total & 344 & 128 & 472 \\
& 72.88 & 27.12 & 100.00 \\
\hline
\end{tabular}

Al revisar en la muestra el tipo de familia en el grupo de adolescentes con violencia y en el grupo sin violencia, predominó la familia nuclear $(54.7 \%)$ y la extensa (23.4\%), siendo más bajos porcentajes la familia monoparental (15\%) y reconstituida $(6.5 \%)$. Es decir que la presencia de ambos padres es la que predomina en la muestra total. Así mismo al revisar el tipo de violencia, según la declaración de los y las adolescentes víctimas, se observa que la violencia psicológica ocupa porcentualmente el primer lugar (67.7\%), seguido por la violencia física $(22.2 \%)$, asimismo la violencia económica y sexual, porcentualmente tienen presencia de 16.7 y 1.5 por ciento respectivamente.

\section{Técnicas e instrumentos de recolección de datos}

Para el presente estudio la aplicación de los instrumentos fue de manera grupal a los alumnos de tercer grado de secundaria, en las Instituciones Educativas Estatales seleccionadas previamente, distribuidas de acuerdo a las zonas urbanas marginales. Los instrumentos que a continuación se describen fueron previamente adaptados lingüísticamente. 
Rosa Huerta, Jaime Aliaga, Emma Campos, Natalia Ramírez, Renato Santiváñez, Jessica Ramos, Lourdes Murillo,

César Falcón, Nohely Treneman, María Tejada, Miriam Castro, Pedro Pérez

\section{Instrumentos:}

1. Ficha de datos sociodemográficos: Elaborada por el investigador. Aplicada de manera grupal. Permite obtener datos como lugar de procedencia, sexo, edad, existencia o no violencia intrafamiliar, el tipo de familia, así como los tipos de violencia en los que se encuentran involucrados.

2. Cuestionario de Depresión de Kovacs. Es un instrumento de indagación aplicable a sujetos de 8 a 17 años. Consta de 27 reactivos, cada uno de ellos está estructurado por tres enunciados identificados con las letras A, B y C, de los cuales el sujeto tiene que seleccionar el que mejor describa la forma en que se ha sentido las dos últimas semanas. Este inventario evalúa un rango de síntomas depresivos que incluyen disturbios en el humor, falta de entusiasmo, funciones vegetativas y dificultades en las relaciones interpersonales. El punto de corte para considerar la presencia de depresión es de 15 puntos. En nuestro medio el CDI fue estandarizado por Ludmila Reátegui (1994), obteniendo una confiabilidad con alfa de Cronbach de 0.81. Ramirez, R. (2009) en Cajamarca, realiza una adaptación del instrumento obteniendo en el análisis total de la prueba un Alfa de Cronbach de 0.79. Para el presente estudio los ítems presentan validez de construcción, encontrándose un coeficiente de consistencia interna Alpha de Cronbach de 0.79 que indica que el cuestionario mide dentro de los márgenes de error permitido.

3. Inventario Multicultural de la Expresión de la Cólera-Hostilidad (IMECH), es un instrumento que permite la medición de la cólera y sus características. En nuestro medio fue adaptada por Nelly Ugarriza (1998) con adolescentes universitarios. Ugarriza se basó en la versión latinoamericana del STAXI, el ML-STAXI, el cual fue adaptado por Moscoso y Rehiser. EL IMECH permite detallar la presencia de sentimientos de cólera y, a su vez, precisar si estos se tratan de una emoción que sienten las personas ante determinados eventos (Cólera Estado), o si constituye un rasgo de su personalidad (Cólera Rasgo). Asimismo, permite conocer cómo es que los sujetos manejan la cólera y la expresión de la misma, si es que tiende a guardarse esos sentimientos (Cólera Contenida) o si por el contrario, tiende a expresarlos hacia afuera arremetiendo contra personas u objetos (Cólera Manifiesta). Asimismo, conocer el modo en que los individuos controlan esta emoción (Control de la Cólera de forma manifiesta o contenida) (Ugarriza, 1998). Cuenta con 44 ítems que se encuentran distribuidos en seis escalas, dos subescalas y una escala que posee las escalas que se refieren a Cólera Contenida, Manifiesta y Control de la misma. Los ítems son respondidos en una escala de tipo Likert con cuatro opciones de respuesta. Puede ser administrado en adolescentes desde los 13 años hasta la edad adulta requiriendo un nivel de lectura acorde al sexto grado de primaria. El tiempo de aplicación es de 15 minutos aproximadamente. En lo que respecta a los puntajes, se obtiene por la sumatoria de los ítems marcados en cada escala y subescala. El puntaje total de la Expresión de la Cólera, se consigue de los 24 ítems que corresponden a las escalas de Cólera Manifiesta, 
DEPRESIÓN Y CÓLERA-HOSTILIDAD EN ALUMNOS VICTIMAS DE VIOLENCIA INTRAFAMILIAR...

Cólera Contenida, y Control de la Cólera Contenida y Manifiesta. Ugarriza en cuanto a la confiabilidad, estudió la consistencia interna a través del Alfa de Cronbach para cada área y prueba global, obtiene niveles satisfactorios para los tres inventarios, y se encontró en el IMECH coeficientes en un rango de 0.64 y 0.93. En el presente estudio respecto al IMECH se encontró un Alpha de Cronbach de 0,87 y 0.82 para Cólera Estado y Cólera Rasgo respectivamente y para Expresión de Cólera (suma de Cólera Manifiesta, Cólera Contenida, y Control de la Cólera Contenida y Manifiesta) un Alpha de Crombach de 0.75 que indica consistencia interna importante y que el cuestionario mide, también, dentro de los márgenes de error permitidos.

\section{RESULTADOS}

1. Relación entre la depresión y la expresión de cólera-hostilidad en alumnos de cuarto grado de secundaria con y sin violencia intrafamiliar de Instituciones Educativas Estatales de zonas urbano marginales de la ciudad de Lima en la muestra general; los datos obtenidos de ambas variables se correlacionaron con el coeficiente Rho de Spearman encontrando relaciones significativas y positivas entre los indicadores de depresión con las dimensiones de la cólera-hostilidad:

- Existe una relación positiva y significativa de 0.41 entre depresión y Cólera Estado, que nos dice que si se incrementa una de ellas también se incrementará la otra, en ambos sentidos es negativo para la salud mental del adolescente pudiendo afectarla de manera crónica o puede ser capaz de agredir con violencia y provocar daños de importancia para sí mismo y su entorno

- Por otra parte, la depresión con la Cólera Rasgo se mantiene en una correlación menor, pero es significativa, y deja entrever que si se incrementa una de las variables, de la misma forma se corre el riesgo de incrementar la otra.

- Finalmente la Expresión de la Cólera presenta menos correlación que las dos anteriores con la depresión, pero el coeficiente hallado es significativo, lo que indica riesgo latente porque la muestra examinada tiende a controlar su cólerahostilidad, hecho que puede retornar de forma agresiva intransitivamente facilitando la cronicidad de la depresión, estos datos pueden verse en la siguiente tabla, concluyendo que existe relación significativa entre la depresión y la cólera- hostilidad en la muestra examinada constituida por adolescentes víctimas y no de violencia familiar.

Tabla 3. De Coeficientes de correlación de la depresión con las dimensiones de la cólerahostilidad.

\begin{tabular}{ccccc}
\cline { 3 - 5 } & & Cólera estado & Cólera Rasgo & $\begin{array}{c}\text { Expresión de } \\
\text { Cólera }\end{array}$ \\
\hline \multirow{2}{*}{ Depresión } & Coeficiente de correlación & 0.409 & 0.379 & 0.188 \\
& Sig. (bilateral) & 0.000 & 0.000 & 0.000 \\
& $\mathrm{~N}$ & 472 & 472 & 472 \\
\hline
\end{tabular}


Rosa Huerta, Jaime Aliaga, Emma Campos, Natalia Ramírez, Renato Santiváñez, Jessica Ramos, Lourdes Murillo,

César Falcón, Nohely Treneman, María Tejada, Miriam Castro, Pedro Pérez

2. Relación entre la depresión y la expresión de cólera-hostilidad en alumnos de cuarto grado de secundaria con y sin violencia intrafamiliar de Instituciones Educativas Estatales de zonas urbano marginales de la ciudad de Lima; los datos obtenidos se correlacionaron con el coeficiente Rho de Spearman, encontrando relaciones positivas entre los indicadores de depresión con las dimensiones de Cólera Estado, Cólera Rasgo y Expresión de Cólera:

- Al analizar los datos en el grupo de adolescentes con violencia intrafamiliar se encuentra una relación significativa (0.00) de 0.43 y 0.40 , entre depresión y cólera estado y entre depresión y cólera Rasgo. En el grupo sin violencia también encontramos una relación de 0.29 y 0.20 , la cual es menor y significativa al 0.05 . Tales resultados nos permiten reforzar lo hallado en la muestra total de una relación positiva entre ambas variables, agravada por la presencia de violencia familiar.

- En lo referente a la expresión de cólera se halló relación entre depresión y expresión de cólera en el grupo de adolescentes con violencia intrafamiliar, no así en el grupo sin violencia. Ello nos indica que el estar inserto en una familia donde hay violencia hace propicio para que el adolescente tenga menor control en la expresión de su cólera pudiéndolo conducir a actos violentos.

Tabla 4. Correlación entre la depresión con la Cólera Estado, Cólera Rasgo y Expresión de Cólera

\begin{tabular}{|c|c|c|c|c|}
\hline & & $\begin{array}{l}\text { Cólera } \\
\text { Estado }\end{array}$ & $\begin{array}{l}\text { Cólera } \\
\text { Rasgo }\end{array}$ & $\begin{array}{l}\text { Expresión de } \\
\text { colera }\end{array}$ \\
\hline \multirow{3}{*}{$\begin{array}{l}\text { Adolescentes con violencia } \\
\text { Depresión }\end{array}$} & $\begin{array}{l}\text { Correlacion Rho de } \\
\text { Spearman }\end{array}$ & $0.43 * *$ & $0.40 * *$ & $0.21 * *$ \\
\hline & Sig. (Bilateral) & 0.00 & 0.00 & 0.00 \\
\hline & $\mathrm{N}$ & 246 & 346 & 346 \\
\hline \multirow{3}{*}{$\begin{array}{l}\text { Adolescentes sin violencia } \\
\text { Depresión }\end{array}$} & $\begin{array}{l}\text { Correlacion Rho } \\
\text { de Spearman }\end{array}$ & $0.29 * *$ & $0.20 *$ & 0.05 \\
\hline & Sig. (Bilateral) & 0.00 & 0.02 & 0.59 \\
\hline & $\mathrm{N}$ & 126 & 126 & 126 \\
\hline
\end{tabular}

** La correlación es significativa al nivel 0,01 (bilateral).

* La correlación es significativa al nivel 0,05 (bilateral).

3. Diferencias en la presencia de depresión y cólera-hostilidad según el sexo; el presente análisis efectuado también con alumnos de cuarto grado de secundaria con y sin violencia intrafamiliar de Instituciones Educativas Estatales de zonas urbano marginales de la ciudad de Lima, para lo cual se ha contrastado utilizando el análisis U de Mann Whitney, con el objeto de conocer si estas variables difieren según el sexo en adolescentes víctima y no de violencia familiar encontrando:

a. En la muestra general son las mujeres quienes presentan mayor número promedio de indicadores de depresión (15.466); ahora bien, cuando hay 
violencia intrafamiliar, los niveles de depresión se incrementan en las mujeres (16.4) a diferencia de los hombres (14.2), hecho que no se encuentra cuando no ocurre violencia familiar.

b. La Cólera Estado tiende a ser mayor en las mujeres adolescentes víctimas de violencia familiar y se torna menor cuando el ambiente familiar es sin violencia.

c. La Cólera Rasgo suele ser mayor en las mujeres que en lo varones.

d. La Expresión de Cólera-hostilidad es una constante cuando hay violencia tanto en hombres como en mujeres y se aminora cuando no la hay. Tal como puede verse en la siguiente tabla de estadísticos descriptivos previos a la contrastación.

Tabla 5. Descripción de los datos obtenidos en las variables depresión y dimensiones de la cólera-hostilidad según el sexo en adolescentes víctimas y no de violencia familiar.

\begin{tabular}{lccccccc} 
& Sexo & N & $\begin{array}{c}\text { Rango } \\
\text { promedio }\end{array}$ & $\begin{array}{c}\text { Suma de } \\
\text { rangos }\end{array}$ & $\begin{array}{c}\text { Media } \\
\text { General }\end{array}$ & $\begin{array}{r}\text { Media CON } \\
\text { VIOLENCIA }\end{array}$ & $\begin{array}{r}\text { Media SIN } \\
\text { VIOLENCIA }\end{array}$ \\
\hline DEPRESIÓN & Mujer & 236 & 255.54 & 60306.50 & 15.466 & 16.432 & 12.633 \\
& Hombre & 236 & 217.46 & 51321.50 & 13.970 & 14.244 & 13.294 \\
& Total & 472 & & & & & \\
CÓLERA & Mujer & 236 & 242.27 & 57176.50 & 13.661 & 14.278 & 11.850 \\
ESTADO & Hombre & 236 & 230.73 & 54451.50 & 13.492 & 13.714 & 12.941 \\
& Total & 472 & & & & & \\
CÓLERA & Mujer & 236 & 248.26 & 58590.00 & 20.025 & 20.949 & 17.317 \\
RASGO & Hombre & 236 & 224.74 & 53038.00 & 19.047 & 19.393 & 18.191 \\
& Total & 472 & & & & & \\
EXPRESIÓN & Mujer & 236 & 232.57 & 54886.00 & 34.958 & 34.523 & 36.233 \\
DE COLERA & Hombre & 236 & 240.43 & 56742.00 & 35.318 & 35.131 & 35.779 \\
& Total & 472 & & & & & \\
\hline
\end{tabular}

e. Al contrastar los rangos promedios se encuentra diferencias significativas según el sexo en los adolescentes víctimas y no de violencia familiar. Se observa que en la depresión las mujeres víctimas de violencia presentan significativamente mayor número de indicadores de depresión que los varones.

En la Cólera Estado también son las mujeres víctimas de violencia quienes presentan significativamente características más definidas en esta variable. 
Rosa Huerta, Jaime Aliaga, Emma Campos, Natalia Ramírez, Renato Santiváñez, Jessica Ramos, Lourdes Murillo,

César Falcón, Nohely Treneman, María Tejada, Miriam Castro, Pedro Pérez

En la Cólera Rasgo son las mujeres víctimas de violencia quienes presentan mayor rango promedio que los hombres.

En la Expresión de Cólera-hostilidad tanto las mujeres como los varones presentan este indicador.

Tabla 11. De contrastación de la depresión y dimensiones de cólera-hostilidad según el sexo en adolescentes víctimas y no de violencia familiar.

\begin{tabular}{ccccc} 
& Depresión & Cólera-Estado & Cólera-Rasgo & Exp. Cólera-Hostilidad \\
\hline U de Mann-Whitney & 15999 & 16949.5 & 15222 & 19003.5 \\
Z & -4.43 & -3.76 & -5.03 & -2.14 \\
$\begin{array}{c}\text { Sig. asintót. } \\
\text { (bilateral) }\end{array}$ & 0.00 & 0.00 & 0.00 & 0.03 \\
\hline
\end{tabular}

\section{INTERPRETACIÓN DE DATOS}

Cuando la adolescencia, etapa de vida de significativos cambios, está influenciada por situaciones que producen inestabilidad,como la violencia intrafamiliar, se incrementa el riesgo de la conducta suicida, resultado de la depresión (Barna, Concha, Florenzano, Gonzales, Horwitz, Maddaleno, Romero, Salazar, Segovia, Valdés, Ventuini, Villaseca y Ringeleng, 1986). En el presente estudio al analizar la muestra general se encuentra que las adolescentes mujeres presentan mayor número promedio de indicadores de depresión (15.46) y se incrementa cuando hay violencia intrafamiliar (16.4) a diferencia de los hombres (14.2), hecho que no se encuentra cuando no hay violencia familiar. Estos datos nos indican un alto riesgo para la salud mental de la adolescente, como también lo manifiestan Vargas, Tovar y Velarde (2010) en su estudio sobre prevalencia y factores asociados con el episodio depresivo en adolescentes de Lima Metropolitana y Callao, quienes encontraron una prevalencia actual del episodio depresivo en adolescentes de $8.6 \%$, siendo mayor en el grupo etario de 15 a 17 años $(12.1 \%)$, con prevalencia en las mujeres (13.4\%) a diferencia de los varones (3.9\%). Asimismo, en cuanto a la prevalencia de episodio depresivo en adolescentes según sensación de soporte familiar y social, se encontró que si esta disminuía, la prevalencia de episodio depresivo aumentaba (de $6.2 \%$ a $14 \%$ ). El ambiente familiar constituye fuente fundamental para el desarrollo o no de este transtorno, así lo observamos en el estudio realizado por el MINSA (2009) en la población adolescente. En este jse señala que entre los principales factores por cuales los y las adolescentes intentan suicidarse son los problemas con los padres y parientes.

$\mathrm{Al}$ revisar las dimensiones de la cólera, la vivencia de esta emoción se vuelve crucial en esta etapa y su manejo una tarea de desarrollo importante. Por ello, aquellos que no logren manejar dicha emoción están en riesgo de enfrentar futuros excesos y actitudes que pueden resultar en conductas agresivas, las cuales pueden traducirse en agresión hacia los otros (Papalia y cols., 2002). Así la presencia de depresión y su relación con la cólera-hostilidad en la adolescencia, ponen en evidencia los 
efectos de la violencia intrafamiliar en la salud mental de esta etapa de vida; pues, encontramos en la muestra global al revisar según sexo, que la Cólera Estado tiende a ser mayor en las mujeres adolescentes víctimas de violencia familiar y se torna menor cuando el ambiente familiar es sin violencia, asimismo la cólera rasgo suele ser mayor en las mujeres que en lo varones. Existiendo relación significativa entre la depresión con cólera estado y depresión con Cólera Rasgo especialmente en el grupo de adolescentes víctimas de violencia intrafamiliar 0.43 y 0.40 respectivamente (nivel de significancia .000). Por otro lado se observa que en el grupo de adolescentes sin violencia existe correlación entre depresión y Cólera Estado (0.29) (nivel de significancia 0.01) y entre depresión con Cólera Rasgo (0.20) (nivel de significancia 0.05). Todo lo cual nos permite afirmar que existe una relación positiva entre depresión y Cólera Estado y entre depresión y Cólera Rasgo cuanto se incrementa una de ellas la otra variable también se aumentará, al evaluarla dentro del contexto de violencia intrafamiliar interfiere de manera dramática el desarrollo de la/el adolescente. Hallazgos que coinciden con Suárez \& Menkes (2006), quienes manifiestan que la exposición constante de los jóvenes a la violencia familiar desde temprana edad tiene alcances devastadores en el desarrollo cognitivo, afectivo y de relación, haciéndolos susceptibles a presentar síntomas que van desde dolores de cabeza o estómago, problemas de sueño, estrés y angustia, hasta estados depresivos, psicóticos, presentando bajo rendimiento escolar, problemas de conducta y adicciones. Además de las consecuencias directas, varios estudios encuentran que los hijos repiten los patrones de violencia vividos en sus hogares. Igualmente Chapi (2012) encuentra una relación inversa entre la satisfacción familiar y la Cólera Estado y Cólera Rasgo observándose que la percepción de satisfacción o insatisfacción familiar inciden en la expresión de cólera, es decir ante el incremento de satisfacción familiar en los adolescentes varones y mujeres, disminuirá la cólera que forma parte del rasgo de personalidad y viceversa.

Finalmente, en lo referente a la expresión de cólera que tiene que ver con el control o no de la misma, esta es una constante cuando hay violencia tanto en hombres como en mujeres y se aminora cuando no la hay. Encontrándose relación entre depresión y Expresión de Cólera en el grupo de adolescentes con violencia intrafamiliar, no así en el grupo sin violencia. Una vez más, destacamos que el estar inserto en una familia donde hay violencia hace propicio para que el adolescente tenga menor control en la expresión de su cólera pudiendolo conducir a actos violentos. Como también lo encuentra Chapi (2012), en un estudio con adolescentes, que a mayor satisfacción familiar, menores indicadores de ansiedad y de cólera - hostilidad, a la vez mayor control de emociones coléricas. Observando además diferencias significativas entre varones y mujeres; las adolescentes obtuvieron mayores puntajes promedio en las escalas de ansiedad y en las escalas de cólera - hostilidad, en contraste, los hombres alcanzaron mayores puntajes promedio en satisfacción familiar y en las escalas control de la cólera manifiesta y control de la cólera contenida. Lo cual nos lleva, una vez más, a manifestar que la familia, en la cual están insertas las y los adolescentes es relevante para su desarrollo como etapa de vida y en su salud mental. Por otro lado Bandura (Bandura, \& Ribes, 
Rosa Huerta, Jaime Aliaga, Emma Campos, Natalia Ramírez, Renato Santiváñez, Jessica Ramos, Lourdes Murillo,

César Falcón, Nohely Treneman, María Tejada, Miriam Castro, Pedro Pérez

1975) y Sánchez (2002) afirman que las influencias familiares principalmente de padres y las personas mayores, mediante sus conductas de sobre exigencia y dominación tienden a establecer pautas hostiles y agresivas en los menores de la familia, hecho que repiten los niños y adolescentes con sus pares. Respecto a las relaciones familiares y la cólera-hostilidad Allen et al. (1996), establecen relación significativa entre el comportamiento hostil, determinado por los conflictos en la relación padres e hijos, y el desarrollo de la autonomía; el no establecer normas en el hogar podrían generalizar actitudes negativas de las normas sociales que devendrían en presentación de agresividad, cólera y hostilidad en jóvenes y adolescentes. Ugarriza (1998) manifiesta que, si el hogar y la escuela no ofrecen condiciones favorables para la sana convivencia entre sus miembros, y más bien se perciben y viven acontecimientos desagradables los adolescentes son quienes principalmente van a querer escapar de esta situación, que al tornarse inevitable o de mal manejo emocional, es probable la presencia de comportamiento hostil y agresivo en ellos con su entorno.

\section{CONCLUSIONES}

- En la muestra total constituida por víctimas y no víctimas de violencia intrafamiliar existe una relación positiva y significativa entre depresión y Cólera Estado de 0.41; entre depresión y Cólera Rasgo de 0.379, indicador de que si se incrementa una de ellas también se incrementará la otra, en ambos sentidos es negativo para la salud mental del adolescente pudiendo afectarla de manera crónica y hacerlo capaz de agredir con violencia y provocar daños de importancia para sí mismo y su entorno.

- En lo que respecta a la Expresión de la Cólera en la muestra general presenta menos correlación que las dos anteriores con la depresión $(\mathrm{r}=0.188, \mathrm{p}=00)$, pero el coeficiente hallado es significativo, lo que indica riesgo latente para controlar la cólera-hostilidad, hecho que puede retornar de forma agresiva intransitivamente facilitando la cronicidad de la depresión.

- Al relacionar los grupos con y sin violencia se encuentra en los adolescentes con violencia intrafamiliar una relación significativa $(\mathrm{p}=0.00)$ de 0.43 y 0.40 , entre depresión y Cólera Estado, y entre depresión y Cólera Rasgo. En el grupo sin violencia una relación de 0.29 y 0.20 , la cual es menor y significativa $(\mathrm{p}=$ 0.05 ). Esta relación positiva entre ambas variables se hace más grave cuando el adolescente es víctima de violencia familiar.

- En lo referente a la Expresión de Cólera se halló relación entre depresión y Expresión de Cólera en el grupo de adolescentes con violencia intrafamiliar, no así en el grupo sin violencia. El estar inserto en una familia donde hay violencia hace propicio para que el adolescente tenga menor control en la expresión de su cólera pudiendolo conducir a actos violentos.

- Al contrastar las diferencias según sexo y ser víctima o no de violencia intrafamiliar, en la muestra general las mujeres presentan más indicadores de 
DEPRESIÓN Y CÓLERA-HOSTILIDAD EN ALUMNOS VICTIMAS DE VIOLENCIA INTRAFAMILIAR...

depresión (15.466); cuando hay violencia intrafamiliar los niveles de depresión se incrementan en las mujeres (16.4) a diferencia de los hombres (14.2), no se encuentran estos indicadores en el grupo sin violencia familiar.

- La Cólera Estado tiende a ser mayor en las mujeres adolescentes víctimas de violencia familiar y es menor en el grupo sin violencia.

- La Cólera Rasgo suele ser mayor en las mujeres con violencia que en lo varones, en el grupo sin violencia no hay diferencias entre mujeres y varones.

- La Expresión de Cólera-hostilidad se encuentra se manera similar cuando hay violencia tanto en hombres como en mujeres y se aminora cuando no la hay.

\section{REFERENCIAS BIBLIOGRÁFICAS}

Acevedo, J. (2008). La cólera en mujeres adolescentes en riesgo de presentar trastornos alimenticios. Tesis para optar por el Título de Licenciatura, Pontificia Universidad Católica del Perú, Lima.

Acosta, M. y Gerena, R. y Montaña, C. (2009). Estudio descriptivo correlacional entre ira y personalidad. Recuperado en www.revistas.unal.edu.co/index.php/ psicologia/article/download/.../1748

American Psychiatric Association (2000). Diagnostic And Statiscal Manual Of Mental Disorders: DSM-IV-TR. 4TH ED, Tex Rev. Washington, D.C: American Psychiatric Association.

Allen, J., Hauser, S., O'Connor, T., Bell, K. \& Eickholt, C. (1996). The connection of observed hostile family conflict to adolescents developing autonomy and relatedness with parents. Development and Psychopathology, 8, 425-442.

Alonso-Fernández, F. (1998). Cuestionario estructural tetradimensional para la depresión. Madrid: Editorial TEA Ediciones S.A.

Arias, I., et al. (1999). Prevalence and correlates of physical aggression during courtship. Journal of Interpersonal Violence, 2, 82-90.

Bandura, A. \& Ribes, E. (1975). Modificación de conducta. México D. F.: Trillas.

Barna R., Concha, I., Florenzano, R., Gonzales, A., Horwitz, N., Maddaleno, M., Romero, M., Salazar, D., Segovia, I., Valdés, M., Ventuini, G., Villaseca P., Ringeleng, I., (1986). Salud familiar. Santiago de Chile: Ed. Corporación de Promoción Universitaria.

Chapi, J. (2012).Satisfacción familiar, ansiedad y cólera-hostilidad en adolescentes de dos instituciones educativas estatales de Lima. Tesis para optar el Título de Psicólogo. Universidad Nacional Mayor de San Marcos. 
Rosa Huerta, Jaime Aliaga, Emma Campos, Natalia Ramírez, Renato Santiváñez, Jessica Ramos, Lourdes Murillo,

César Falcón, Nohely Treneman, María Tejada, Miriam Castro, Pedro Pérez

Corsi y colbs.(1997). Violencia Familiar. Una mirada interdisciplinaria sobre un grave problema social. Buenos Aires: Paidós.

Gotlib Ih, Lewinsohn Pm, Seeley Jr. (1995). Symptoms Versus a Diagnosis Of Depression: Difference In Psychosocial Functioning. J Consult Clin Psychol:;63(1):90-100.

Huerta, R. et al. (2014). Depresión y ansiedad en mujeres en situación de maltrato en la relación de pareja según tipo de convivencia en zonas urbano marginales de la ciudad de Lima. Theorema. Segunda Época. Universidad Nacional Mayor de San Marcos, 1(1), 123-136.

Instituto Nacional de Salud Mental Honorio Delgado- Hideyo Noguchi (2013). www. insm.gob.pe/oficinas/comunicaciones/notasdeprensa/2013/np.htm

Instituto Especializado de Salud Mental Honorio Delgado - Hideyo Noguchi. (2002). Estudio Epidemiológico Metropolitano en Salud Mental 2002. Informe General. Anales de Salud Mental 18(1-2):1-197.

Izard, C. (1991). Psychology of Emotions. New York, USA: Plenum.

Fernández - Abascal, E. \& Martín, M. (1994). Ira y hostilidad en los trastornos coronarios. Anales de Psicología, 10 (2), 177-188.

Flores, T, y Schirmer, J. (2006). Violencia intrafamiliar en la adolescencia. Revista Latino-am Enfermagem. Recuperado en www.eerp.usp.br/rlae

Moscoso, M. (1999). Medición de la Expresión de la Cólera y Hostilidad. Revista de la Facultad de Psicología de la Universidad Mayor de San Marcos, 3(4), 169-188.

Moscoso, M. (2000). Estructura Factorial del Inventario Multicultural Latinoamericano de la Expresión de la cólera y la hostilidad. Revista Latinoamericana de Psicología, 32(2), 321-343.

Muro, M. (2010). Cólera y acoso escolar en un grupo de adolescentes de un colegio estatal en Lima metropolitana. Tesis para optar el Título de Licenciatura. Pontificia Universidad Católica. Lima. Perú.

Papalia, D., Wendkos, S. y Duskin, R. (2002). Psicología del desarrollo: de la infancia a la adolescencia (9). México D.F., México: Mc Graw-Hill Interamericana.

Sánchez, A. (2002). Psicología social aplicada. Madrid: Pearson Educación.

Silva, I. (2008) La adolescencia y su interrelación con el entorno. Recuperado en www.injuve.es/.../LA\%20ADOLESCENCIA $\% 20 y \% 20 \% 20$ entorno_com... 
DEPRESIÓN Y CÓLERA-HOSTILIDAD EN ALUMNOS VICTIMAS DE VIOLENCIA INTRAFAMILIAR...

Suárez L \& Menkes C (2006) La violencia familiar contra adolescentes. Rev Saúde Pública; 40(4):611-9. Recuperado en www.scielo.br/pdf/rsp/v40n4/09.pdf

Spielberger, C y Reheiser, E. (2009). Assesment of Emotions: Anxiety, Anger, Depression and Curiosity [Versión Electrónica]. International Association of Applied Psychology.1 (3), 271-302.

Ugarriza, N. (1998). Normalización del Inventario Multicultural de la Expresión de la Cólera-Hostilidad en estudiantes universitarios. IPSI Revista de Investigación en Psicología, 1(1), 59-88.

Vargas, H., Tovar, H. y Valverde, J. (2010) Prevalencia y factores asociados con el episodio depresivo en adolescentes de Lima Metropolitana y Callao Revista Peruana de Epidemiología. Vol.14(2).

Veytia, M., et al. (2012). Depresión en adolescentes: El papel de los sucesos vitales estresantes. Recuperado en www.scielo.org.mx/scielo. php?pid=S0185-33252012000100006 